\title{
Protocol and Baseline Data on Renal Autologous Cell Therapy Injection in Adults with Chronic Kidney Disease Secondary to Congenital Anomalies of the Kidney and Urinary Tract
}

\author{
Joseph Stavas $^{a} \quad$ Maria Diaz-Gonzalez de Ferris $^{\mathrm{b}} \quad$ Ashley Johns $^{\mathrm{a}}$ Deepak Jain ${ }^{\mathrm{a}}$ \\ Tim Bertram ${ }^{\mathrm{a}}$ \\ aProKidney, Grand Cayman, George Town, Cayman Islands; ${ }^{b}$ Department of Pediatrics, The University of North \\ Carolina at Chapel Hill, Chapel Hill, NC, USA
}

\section{Keywords}

CAKUT in adults · Chronic kidney disease · Autologous cell therapy · Preliminary data

\begin{abstract}
Background: Advanced cell therapies with autologous, homologous cells show promise to affect reparative and restorative changes in the chronic kidney disease (CKD) nephron. We present our protocol and preliminary analysis of an IRBapproved, phase I single-group, open-label trial that tests the safety and efficacy of Renal Autologous Cell Therapy (REACT; NCT 04115345) in adults with congenital anomalies of the kidney and urinary tract (CAKUT). Methods: Adults with surgically corrected CAKUT and CKD stages 3 and 4 signed an informed consent and served as their "own" baseline control. REACT is an active biological ingredient acquired from a percutaneous tissue acquisition from the patient's kidney cortex. The specimen undergoes a GMP-compliant manufacturing process that harvests the selected renal cells composed of progenitors for renal repair, followed by imageguided locoregional reinjection into the patient's renal cortex. Participants receive 2 doses at 6-month intervals. Primary outcomes are stable renal function and stable/im-
\end{abstract}

proved quality of life. Additional exploratory endpoints include the impact of REACT on blood pressure, vitamin D levels, hemoglobin, hematocrit and kidney volume by MRI analysis. Results: Four men and 1 woman were enrolled and underwent 5 cell injections. Their characteristics were as follows: mean 52.8 years (SD 17.7 years), 1 Hispanic, 4 non-Hispanic, and 5 white. There were no renal tissue acquisition, cell injection, or cell product-related complications at baseline. Conclusion: REACT is demonstrating feasibility and patient safety in preliminary analysis. Autologous cell therapy treatment has the potential to stabilize or improve renal function in CAKUT-associated CKD to delay or avert dialysis. Patient enrollment and follow-up are underway.

(c) 2021 S. Karger AG, Basel

\section{Introduction}

The global prevalence of chronic kidney disease (CKD) is approximately $10 \%$ of the population, with regional and race/ethnicity differences. The most common etiologies of CKD among adults are diabetes, hypertension, glomerular disease, and less commonly congenital anomalies of the kidney and urinary tract (CAKUT). CAKUT karger@karger.com

(C) 2021 S. Karger AG, Basel

www.karger.com/bpu

Karger"
Joseph Stavas

Cell Biotechnology, ProKidney

8020 Arco Corporate Drive

Suite 118, Raleigh, NC 27617 (USA)

joseph.stavas@prokidney.com 

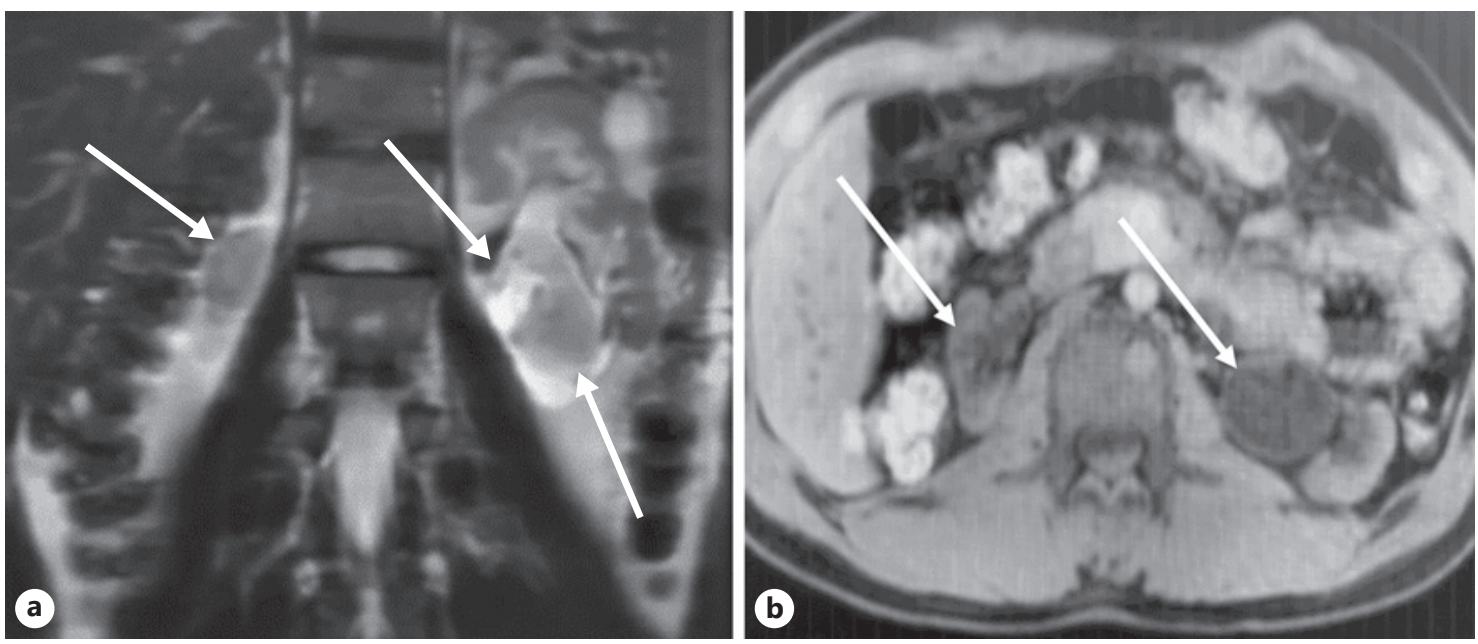

Fig. 1. CAKUT MRI: coronal T2 and axial T1 (a, b) weighted MRI shows dilated central collecting system and asymmetric development of the left kidney with thinning of the lateral cortex (arrows). The right kidney is atrophic with a large bowel filling at the renal fossa. Renal volume obtained from the left kidney to determine dose. CAKUT, congenital anomalies of the kidney and urinary tract; MRI, magnetic resonance imaging.

is the most common cause of pediatric-onset CKD, accounting for approximately $50 \%$ of cases $[1,2]$.

CAKUT is the result of a spectrum of defects in the kidneys and outflow tracts which include the ureters, bladder [3], and urethra, and the prevalence of CAKUT has been estimated to be $4-60$ per 10,000 births, depending on the registry with some ethnic differences [4]. CKD is a condition with unidirectional progression to endstage renal disease (ESRD). In the USRDS, adolescents with ESRD have a 10-year survival of $>85 \%$ [5].

While patients with CAKUT experience slower CKD progression compared to those with glomerular disease [6], ESRD usually occurs in adolescence and young adulthood. While the survival rate of children with ESRD has improved, mortality remains about 30 times higher than that of healthy peers [5]. In the USA, young adult patients with ESRD die due to cardiovascular causes primarily [7, 8], with greater mortality in black patients [9].

Multiple therapies have entered the developmental trial pipeline for $\mathrm{CKD}$; however, most target biochemical or genetic markers for various disease pathways [9-13]. Advanced cell therapies with autologous, homologous cells have shown promise to affect reparative and restorative changes in the CKD nephron and improve glomerulartubular function in animal data [13] and phase I and ongoing phase II trials in adult diabetic patients with CKD. This manuscript describes our protocol trial and a preliminary analysis of a phase I single-group open-label trial testing the safety and efficacy of Renal Autologous Cell
Therapy/Neo-Kidney Augment (REACT ${ }^{\mathrm{TM}}$; NCT 04115345) among adults with CAKUT-related CKD.

\section{Methods}

\section{Intervention}

The active biological ingredient of REACT is acquired from a percutaneous tissue acquisition from the patient's kidney cortex. The kidney tissue undergoes a Good Manufacturing Practicescompliant process that harvests the selected renal cells (SRC) composed of progenitors for renal repair. Cells are held frozen in liquid nitrogen for future use. Prior to reinjection, SRCs are formulated in a thermolabile gelatin-based hydrogel concentration of $100 \times 10^{6}$ cells $/ \mathrm{mL}$ and injected into the kidney cortex of the donor, with a volume-based dose determined by a noncontrast magnetic resonance imaging (MRI) volumetric analysis of the kidneys (Fig. 1). CAKUT parenchyma and collecting system anatomy is heterogeneous, and optimum injection sites are determined by ultrasound and CT to ensure precision placement in the cortex region (Fig. 2). The cell integration process postinjection into the patient is depicted in Figure 3. Following the REACT injection, the patients return for serial clinical visits and laboratory measurements. Participant timeline from trial entry and tissue acquisition through REACT manufacturing and reinjection is depicted in Figure 4. The patients receive 2 doses administered at 6-month intervals, and each patient serves as their "own" control.

\section{Patients}

The recruitment goal is 15 subjects. Major inclusion criteria include females or males aged 18-65 years with a documented history of a surgically corrected CAKUT and an estimated glomerular filtration rate (eGFR) of $14-50 \mathrm{~mL} / \mathrm{min} / 1.73 \mathrm{~m}^{2}$ at the time of 


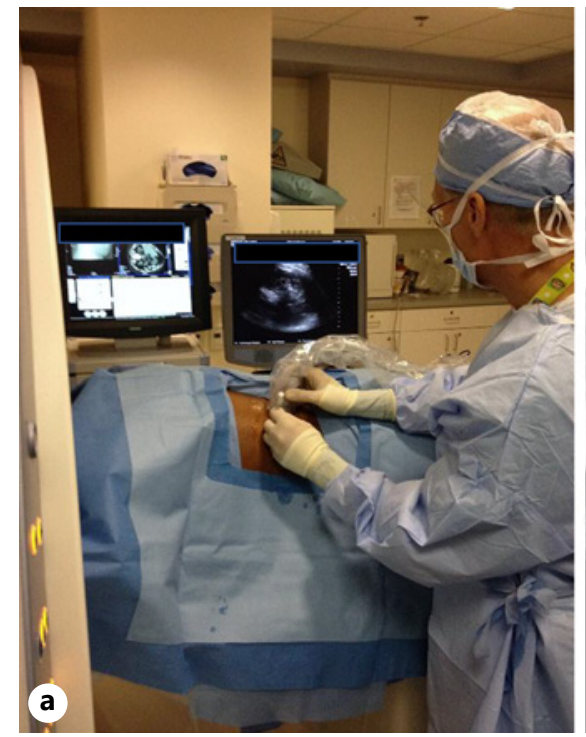

Fig. 2. a Cell injection: percutaneous CT and ultrasound-guided needle insertion into the renal cortex assures precision delivery of REACT and allows real-time evaluation of injection-related complications. b Axial CT image shows heterogeneous CAKUT morphology with needle system entering the capsule and cortex area

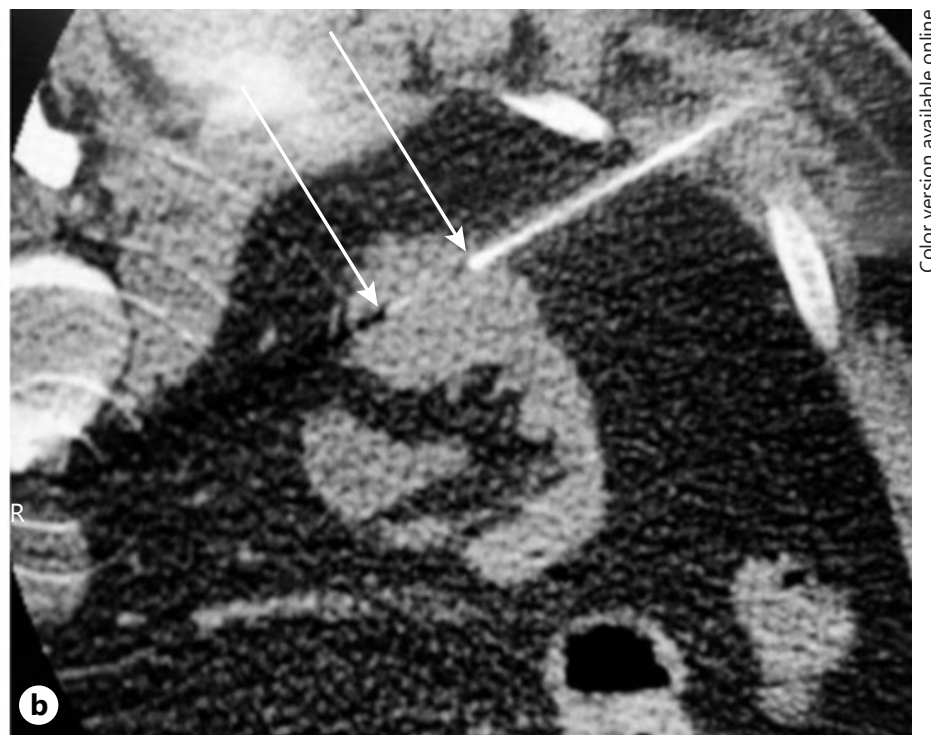

for autologous cell injection (arrows). The small diameter needle platform reduces risks of bleeding and pain. REACT, Renal Autologous Cell Therapy; CAKUT, congenital anomalies of the kidney and urinary tract.
Fig. 3. Autologous cell mechanism of action: cell restoration occurs following REACT injection into the cortex by chemotactic migration to the damaged nephron unit, including tubules in the medullary kidney. Integration into the glomerulus and tubules ensues with cytokine-mediated repair of renal function. (Figure used with permission from Nature.) REACT, Renal Autologous Cell Therapy.

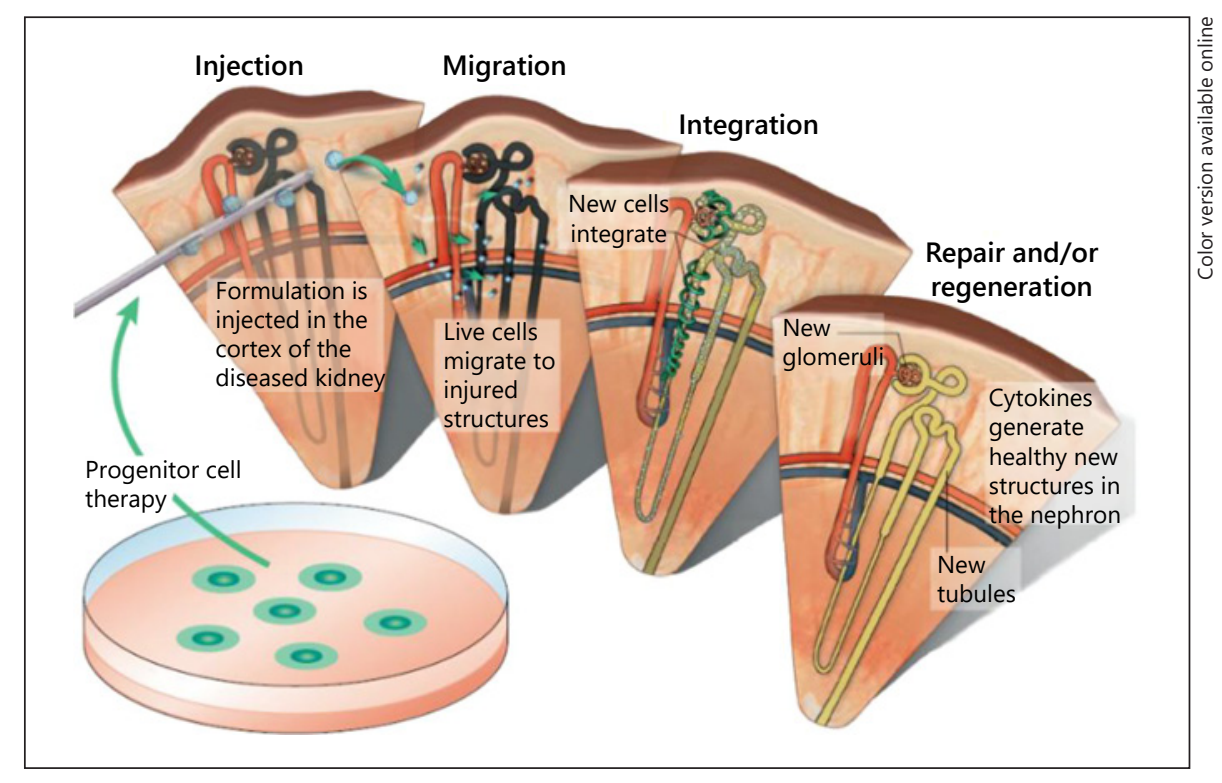

Major exclusion criteria are a history of renal transplantation, grade 4-5 hydronephrosis, uncorrected vesicoureteral reflux (VUR), thinned renal cortex disallowing safe biopsy and cell injection, acute kidney injury in last 3 months, allergy to hydrogel formulation constituents, recent urinary tract infections, renal tumors, and cysts or other anatomic masses. Contraindications also include significant medical problems (cardiac, pulmonary, or hepatic conditions), immunocompromised, and pregnancy. 
Fig. 4. Subject timeline from trial entry and tissue biopsy through REACT manufacturing and reinjection. REACT, Renal Autologous Cell Therapy.

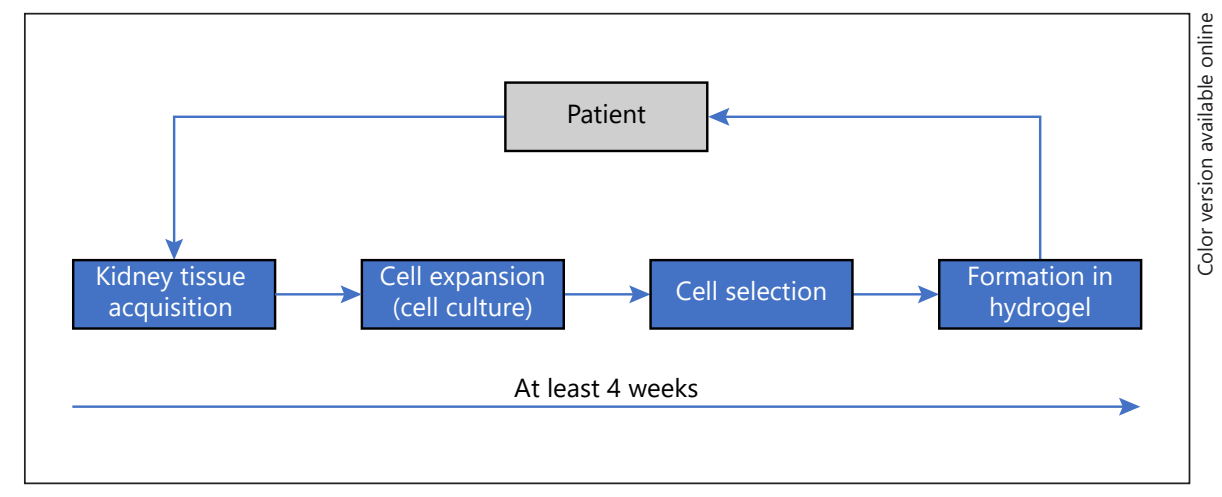

The primary endpoints are improved renal function based upon change in eGFR total slope by CKD-EPI and serum creatinine measurements and incidence of adverse events out to 24 months postinjection. Additional exploratory endpoints are the impact of REACT ${ }^{\mathrm{TM}}$ on urinary protein, blood pressure, vitamin D levels, hemoglobin and hematocrit, and kidney volume assessed by MRI.

\section{Results}

We have enrolled 5 patients to date with the following baseline characteristics: 1 woman and 4 men; 5 Caucasians (1 Hispanic and 4 non-Hispanic); mean age 52.8 years (SD 17.7 years; range 22-60 years). The cause of CKD included VUR in all, and one of the men had surgically corrected posterior urethral valves. Comorbidities and their characteristics include the following: all had hypertension and 3 had hyperlipidemia. The woman in our cohort had a mildly elevated baseline blood pressure at $144 / 88$, and all men were normotensive. The cohort's mean estimated glomerular filtration rate by CKD-EPI was 29.5 (range 19-35) $\mathrm{mL} / \mathrm{min} / 1.73 \mathrm{~m}^{2}$. The mean quality of life score was 81.25 (range 75-100). The mean kidney weight based on MRI measurements was $154 \mathrm{~g}$ (SD $72.35 \mathrm{~g}$; range 95-272 g). The mean calculated volume for injection was $2.3 \mathrm{~mL}$ (SD 0.91, range 3-8 mL). Six depositions were injected in the 5 patients, as one of the cohorts required 2, with a mean injection volume of $2.3 \mathrm{~mL} / \mathrm{de}$ position (SD $0.91 \mathrm{~mL}$; range $1.5-3.5 \mathrm{~mL}$ ). The mean sedation time was $44.2 \mathrm{~min}$ (SD $34.7 \mathrm{~min}$; range $20-105 \mathrm{~min}$ ), and the mean injection time was $19.6 \mathrm{~min}$ (SD18.61 min; range $7-56 \mathrm{~min})$.

The 5 enrolled patients showed an adequate cortex thickness of $>5 \mathrm{~mm}$ for safe left-sided kidney tissue acquistion and reinjection on screening ultrasound and MRI. There was normal cell isolation and expansion for each patient, and 6 cell injections were completed. There have been no biopsy, injection, or cell product-related acute or chronic complications.

\section{Discussion}

In this report, we have documented the feasibility and safety of using REACT, an autologous cell therapy, as a potential cell-based treatment that may durably stabilize renal function in CAKUT-associated CKD. The potential to delay or avert dialysis remains to be demonstrated after long-term follow-up is completed.

CAKUT is diagnosed most often prenatally or during pediatric years with crossover implications into later adulthood. The risks for CKD progression include the onset of comorbidities such as metabolic syndrome, hypertension, and cardiovascular disease [15]. Despite the traditional categorization of patients with CAKUT as being "nonglomerular," glomerular-tubular changes have been described [16].

CAKUT is a complex renal disease presenting with a broad spectrum of heterogeneous phenotypes and monoand polygenetic etiologies that may cause long-lasting parenchymal scarring and nephropathy [3]. Despite surgical correction of the gross collecting system abnormality, these patients may progress to ESRD. While there are few histopathological studies in CAKUT patients, renal parenchymal changes, glomerular changes, overt tubuleinterstitial changes, and fibrosis is noted to occur with tubule-interstitial or reduction in proximal tubule size [16].

Based on our experience with animal histopathological effects [17], we moved to address the CAKUT population in humans. There is a significant unmet need for clinical strategies to improve renal function and delay the 
need for renal replacement therapy in progressive CKD [15].

This current trial uses a percutaneous, image-guided techniques with conscious sedation. The phase I trial of high-risk CKD diabetics who were injected with REACT used a laparoscopically assisted surgical approach and general anesthesia [18]. Furthermore, the cellbased therapeutic could be administered (mean eGFR $<30 \mathrm{~mL} / \mathrm{min}$ ) safely in a small cohort [17]. Ongoing phase II clinical trials are exploring additional safety and efficacy (NCT02836574 and NCT03270956) in stage 3-4 diabetic CKD and provide a platform for autologous cell therapy adaptation in additional CKD etiologies.

CAKUT patients are good candidates for autologous kidney cell therapy to stabilize CKD progression or improve it. Following the REACT injection, there is chemotaxis-regulated migration of cells with integration into the glomerular-tubular unit and rebuilding of cellular elements that restore renal function [13], as depicted in Figure 3. Using autologous/homologous cell therapies for CKD has several advantages. In this therapy, the kidney cells that repair and restore nephron structure and function can be effectively expanded in vitro with Good Manufacturing Practices and maintained for future use, with liquid nitrogen preservation. Regardless of the CKD etiology, the kidney late-stage pathology leads to chronic inflammation and fibrosis with damage to glomeruli and tubules.

Potential complications such as radiation exposure during cell injections are rare due to current dose-reduction strategies with focused imaging of the kidneys and awareness of ALARA (As Low As Reasonably Achievable) algorithms. Other complications such as bleeding are avoided by our strict inclusion criteria for anticoagulation assessment that avoids patients at risk for bleeding and use of atraumatic needles in areas of minimal vascular risk in the kidney. No transfusions have been required postprocedure. Sedation in all patients was well tolerated.

REACT is "accepted as self" by the patient, with no identified immunologic and cell transformation consequences. REACT's active biological ingredient is known to survive in analogous environments from the donor kidney, with a half-life of 6 months. Cell administration methods into the kidney are easily standardized and reproducible with precision placement into the renal cortex using image-guided techniques and small caliber needles to maximize cell depostion in a favorable environment. Procedural injection risks are low and performed in outpatient settings with conscious sedation.

\section{Conclusions}

REACT is demonstrating good feasibility and patient safety at baseline and preliminary analysis. Autologous cell therapy is a novel treatment and has the potential to stabilize renal function in CAKUT-associated CKD and delay or avert dialysis. Patient enrollment is underway.

\section{Acknowledgements}

The authors would like to thank their participants for teaming with them to move this evidence forward and advance this field.

\section{Statement of Ethics}

This trial has been censored by Institutional Review Board approval and participant informed consent.

\section{Conflict of Interest Statement}

Diaz-Gonzalez de Ferris: grants and research support Renal Research Institute, ProKidney. Joseph Stavas, Tim Bertram, Ashley Johns, and Deepak Jain are employees of ProKidney.

\section{Funding Sources}

This research trial is privately funded solely by the sponsor ProKidney. Joseph Stavas, Tim Bertram, Ashley Johns, and Deepak Jain are employed by the sponsor ProKidney Maria DiazGonzalez de Ferris is a consultant of the sponsor ProKidney.

\section{Author Contributions}

Drs. Stavas, Jain, Diaz-Gonzalez de Ferris, and Bertram and Ms. Johns have made substantial contributions to the conception of the manuscript and drafting and revision of content, agreed to be accountable for accuracy and result interpretation, and approved the final version for publication.

References

1 Ferris ME, Mahan JD. Pediatric chronic kidney disease and the process of health care transition. Semin Nephrol. 2009 Jul;29(4): $435-44$

2 Harambat J, van Stralen KJ, Kim JJ, Tizard EJ. Epidemiology of chronic kidney disease in children. Pediatr Nephrol. 2012 Mar;27(3): $363-73$. 
3 Murugapoopathy V, Gupta I. Primer on congenital anomalies of the kidneys and urinary tracts (CAKUT). J Am Soc Nephrol. 2020 May 7;15(5):723-31.

4 Tain YL, Luh H, Lin CY, Hsu CN. Incidence and risks of congenital anomalies of kidney and urinary tract in newborns: a populationbased case-control study in Taiwan. Medicine. 2016;95(5):e2659.

5 Ferris ME, Gipson DS, Kimmel PL, Eggers PW. Trends in treatment and outcomes of survival of adolescents initiating end-stage renal disease care in the United States of America. Pediatr Nephrol. 2006 Jul;21(7):1020-6.

6 Furth SL, Pierce C, Hui WF, White CA, Wong CS, Schaefer F, et al. Estimating time to ESRD in children with CKD. Am J Kidney Dis. 2018 Jun;71(6):783-92.

7 Modi ZJ, Lu Y, Ji N, Kapke A, Selewski DT, Dietrich X, et al. Risk of cardiovascular disease and mortality in young adults with endstage renal disease: an analysis of the US renal data system. JAMA Cardiol. 2019 Apr;4(4): 353-62.

8 Parekh RS, Carroll CE, Wolfe RA, Port FK. Cardiovascular mortality in children and young adults with end-stage kidney disease. J Pediatr. 2002 Aug;141(2):191-7.
9 Torres VE, Chapman AB, Devuyst O, Gansevoort RT, Perrone RD, Koch G, et al. Tolvaptan in later-stage autosomal dominant polycystic kidney disease. N Engl J Med. 2017 Nov;377(20):1930-42.

10 Perkovic V, Jardine MJ, Neal B, Bompoint S, Heerspink HJL, et al. Canagliflozin and renal outcomes in type 2 diabetes and nephropathy. N Engl J Med. 2019 Jun 13;380(24):2295-306.

11 Brenner BM, Cooper ME, de Zeeuw D, Keane WF, Mitch WE, Parving HH, et al. Effects of losartan on renal and cardiovascular outcomes in patients with type 2 diabetes and nephropathy. N Engl J Med. 2001 Sept;345(12): 861-9.

12 Ruiz S, Pergola PE, Zager RA, Vaziri ND. Targeting the transcription factor Nrf2 to ameliorate oxidative stress and inflammation in chronic kidney disease. Kidney Int. 2013; 83(6):1029-41.

13 Kelley R, Bruce A, Spencer T, Werdin E, Ilagan $\mathrm{R}$, Choudhury $\mathrm{S}$, et al. A population of selected renal cells augments renal function and extends survival in the ZSF1 model of progressive diabetic nephropathy. Cell Transplant. 2013;22(6):1023-39.
14 Levey A, Stevens L, Schmid C, Zhang L, Alejandro F, Castro A, et al.; for the Chronic Kidney Disease Epidemiology Collaboration (CKD-EPI). A new equation to estimate glomerular filtration rate. Ann Intern Med. 2009 May 5;150(9):604-12.

15 Fraser SD, Barker J, Roderick PJ, Yuen HM Shardlow A, Morris JE, et al. Health-related quality of life, functional impairment and comorbidity in people with mild-to-moderate chronic kidney disease: a cross-sectional study. BMJ Open. 2020;10(8):e040286.

16 Huang WY, Peters CA, Zurakowski D, Borer JG, Diamond DA, Bauer SB, et al. Renal biopsy in congenital ureteropelvic junction obstruction: evidence for parenchymal maldevelopment. Kidney Int. 2006 Jan 1;69(1):13743.

17 Presnell SC, Bruce AT, Wallace SM, Choudhury S, Genheimer C, Cox B, et al. Isolation, characterization, and expansion (ICE) methods for defined primary renal cell populations from rodent, canine, and human normal and diseased kidneys. Tissue Eng Part C Methods. 2011 Mar;17(3):261-73.

18 Stenvinkel P, Wadström J, Bertram T, Detwiler R, Gerber D, Brismar TB, et al. Implantation of autologous selected renal cells in diabetic chronic kidney disease stages 3 and 4-clinical experience of a "First in Human" study. Kidney Int Rep. 2016 Sept; 1(3):105-13. 